\title{
Influencia del Diseño Micro y Macroscópico de la Región Cervical del Implante en la Preservación de Hueso Marginal Perimplantario: Una Revisión Sistemática
}

\author{
Influence of the Micro and Macroscopic Implant Neck Design \\ in the Preservation of Marginal Bone Level: A Systematic Review
}

\begin{abstract}
Markel Diéguez Pereira*; Gorka Santamaría Arrieta*; Felipe J. Fernández- González ${ }^{* \star *}$; José Antonio Vega Álvarez ${ }^{* * * *}$; Iker Bellanco de la Pinta ${ }^{* *+* *}$; Nerea Martín Blanco* \& Aritza Brizuela Velasco*
\end{abstract}

DIÉGUEZ PEREIRA, M.; SANTAMARÍA ARRIETA G.; FERNÁNDEZ GONZÁLEZ, F. J.; VEGA ÁlVAREZ, J. A.; BELLANCO PINTA, I.; MARTÍN BLANCO N. \& BRIZUELA VELASCO. A. Influencia del diseño micro y macroscópico de la región cervical del implante en la preservación de hueso marginal perimplantario: Una revisión sistemática. Int. J. Odontostomat., 9(1):119-127, 2015.

RESUMEN: El objetivo de esta revisión sistemática fue evaluar los distintos diseños en el cuello del implante (pulido o tratado, con o sin intercambio de plataforma, o con o sin microespiras) y su posible influencia en la pérdida del hueso marginal periimplantario. Se llevo a cabo una revisión sistemática siguiendo la metodología PICO del Centro de Medicina Basada en la Evidencia de la Universidad de Oxford. La pregunta dirigida a la consecución de los objetivos fue: ¿Es el diseño del cuello del implante un factor crítico en la conservación del hueso marginal periimplantario? Se emplearon MeSH keywords específicos en las bases de datos Pubmed y Cochrane. Tres revisores independientes se pusieron de acuerdo en los estudios finalmente incluidos, obteniendo un índice de concordancia kappa de 0,88. De estos, se realizó una evaluación crítica del nivel de evidencia y también del riesgo de sesgo de los RCT mediante la Herramienta Cochrane. Se consultaron 445 artículos por título, incluyendo finalmente un total de 16 artículos a texto completo. La heterogeneidad de estos estudios impidió realizar un meta-análisis. No se encontró evidencia de que una determinada configuración del cuello del implante sea mejor que otra en la conservación del hueso marginal periimplantario y sin embargo, parece que una posición apico-coronal yuxtaósea del cuello pulido del implante respecto a la cresta ósea receptora pudiera ser crítica a ese respecto. No se encontró suficiente evidencia sobre la efectividad de las diferentes configuraciones de la región cervical del implante en la preservación del hueso marginal periimplantario. Son necesarios estudios clínicos controlados y aleatorizados a largo plazo para valorar los efectos de dichas modificaciones.

PALABRAS CLAVE: reabsorción ósea, cuello del implante, pérdida ósea marginal, diseño del cuello del implante, plataforma modificada.

\section{INTRODUCCIÓN}

Uno de los factores más relevantes entre los criterios de éxito en la rehabilitación implantológica es la presencia de un nivel adecuado de hueso periimplantario, evaluado radiográficamente tras la carga protésica (Albrektsson et al., 1986). Tradicionalmente se ha considerado aceptable una pérdida ósea marginal de 1-2 mm durante el primer año de carga y de 0,05-0,2 $\mathrm{mm}$ anuales a partir de éste (Branemark et al., 1977). No obstante, se ha observado que una reabsorción ósea excesiva compromete la supervivencia tanto del implante como de la prótesis sobre él retenida. Los factores involucrados en esta reabsorción

Posgrado de Especialista Universitario en Implantología Oral. Departamento de Estomatología I, Universidad del País Vasco UPV-EHU, Leioa, España.

* Profesor del Posgrado de Especialista Universitario en Implantología Oral. Departamento de Estomatología I. Universidad del País Vasco UPV-EHU, Leioa, España.

*** Profesor Posgrado de Ortodoncia y Ortopedia Dentofacial. Universidad de Oviedo, Oviedo, España.

***** Facultad de Ciencias de la Salud, Universidad Autónoma de Chile, Chile. Catedrático Departamento de Morfología y Biología Celular. Universidad de Oviedo, Oviedo, España.

***** Licenciado en Odontología por la Universidad del País Vasco UPV-EHU, Leioa, España. 
DIÉGUEZ PEREIRA, M.; SANTAMARÍA ARRIETA G.; FERNÁNDEZ GONZÁLEZ, F. J.; VEGA ÁLVAREZ, J. A.; BELLANCO PINTA, I.; MARTÍN BLANCO N. \& BRIZUELA VELASCO. A. Influencia del diseño micro y macroscópico de la región cervical del implante en la preservación de hueso marginal perimplantario: Una revisión sistemática. Int. J. Odontostomat., 9(1):119-127, 2015

son múltiples, siendo el factor biológico comúnmente aceptado como determinante de la presencia de bacterias patógenas responsables de la aparición de periimplantitis (Lang et al., 2011). Por otro lado, diversos estudios experimentales tanto en animales (Isidor, 1996; Miyata et al., 1998, 2000) como in vitro (Rungsiyakull et al., 2011) observan pérdidas de hueso marginal periimplantario como resultado de la aplicación de cargas oclusales excesivas o mal diseñadas.

Otros factores que parecen implicados en la reabsorción ósea periimplantaria son la falta de ajuste entre implante y estructura protésica (Assunçao et al., 2011), la incorrecta colocación tridimensional del implante (Tomasi et al., 2010) o el posicionamiento subcrestal de la plataforma del implante (Grunder et al., 2005).

Aunque el proceso de remodelado que conduce a la reabsorción de hueso marginal periimplantario puede ocurrir en cualquier zona del implante, lo más habitual es que éste se produzca alrededor de su cuello (Adell et al., 1990). Por este motivo se considera que la selección del diseño adecuado del implante en su zona cervical puede ser clave para su supervivencia. A este respecto, Lazzara \& Porter (2006) describieron un método de preservación de hueso marginal consistente en la mecanización de pilares protésicos de un diámetro menor que el de la plataforma de los implantes. Este método, fruto de un hallazgo casual, es lo que se conoce actualmente como intercambio de plataforma o platform switching. Existen varias hipótesis que tratan de explicar el mecanismo por el cual el uso de esta técnica podría disminuir la reabsorción ósea, una de ellas consistiría en la creación de un espacio horizontal alrededor del cual se dispondrían las fibras de tejido conectivo (Hermann et al., 2001; Lazzara \& Porter). También podría explicarse por el desplazamiento del infiltrado inflamatorio, tanto desde el hueso crestal hacia el centro de la plataforma del implante (Abrahamsson et al., 1998), como del área de máximo estrés biomecánico (Maeda et al., 2007; Chang et al., 2010).

La evidencia científica disponible que pueda avalar cualquiera de estas teorías es insuficiente y el conocimiento de los mecanismos de pérdida ósea marginal es aún limitado. El objetivo de esta revisión sistemática fue determinar si el módulo de la cresta del implante influye o no en la pérdida de hueso marginal periimplantario.

\section{MATERIAL Y MÉTODO}

Se efectuó una revisión sistemática de la literatura empleando la sistemática PICO (Patient or Population, Intervention, Control or Comparison y Outcome) del Centro de Medicina Basada en la Evidencia (CEBM) de la Universidad de Oxford. La estrategia de búsqueda incluyó el empleo de $\mathrm{MeSH}$ Keywords específicos, derivados de la pregunta dirigida, en las bases de datos Cochrane y Pubmed desde Enero de 2004 hasta Junio de 2014.

Pregunta dirigida: ¿Es el diseño del cuello del implante un factor crítico en la conservación del hueso marginal perimplantario?

Estrategia de búsqueda: La Tabla I aporta detalles de la estrategia de búsqueda PICO, criterios de inclusión y exclusión, y las bases de datos y revistas indexadas en donde se identificaron los artículos. Esta primera estrategia estuvo diseñada para obtener un número alto de entradas relacionadas, más que una alta precisión. No hubo restricciones de idioma (Tabla I).

\section{Selección de estudios y procedimientos de eva-} luación de su calidad: La búsqueda fue realizada por tres revisores independientes, que pusieron en común sus resultados particulares. La inclusión final de los trabajos se decidió por consenso entre los tres revisores obteniendo un índice de concordancia kappa de 0,88 . Se procedió a su tipificación de nivel de la evidencia en base a la clasificación CEBM de la Universidad de Oxford, así como a los riesgos de sesgo de todos ellos, basados en la Herramienta Cochrane ("Risk of bias"- Cocrane Handbook for Systematic Reviews of Interventions).

Estrategia de obtención de datos: El dato principal que todos los estudios incluidos debían analizar era la pérdida de hueso marginal en diferentes momentos. Los estudios debían incluir la diferencia radiográficamente observada y numéricamente cuantificada entre el nivel de hueso marginal peri-implantario en el inicio y en los diferentes momentos de seguimiento, a partir de una referencia anatómica especificada.

Para cada estudio incluido, se recogieron los datos relativos a los pacientes, implantes y resultados y se usó la escala de calidad Jadad et al. (1996) para evaluar los intervalos de confianza de los estudios incluidos. 
DIÉGUEZ PEREIRA, M.; SANTAMARÍA ARRIETA G.; FERNÁNDEZ GONZÁLEZ, F. J.; VEGA ÁLVAREZ, J. A.; BELLANCO PINTA, I.; MARTÍN BLANCO N. \& BRIZUELA VELASCO. A. Influencia del diseño micro y macroscópico de la región cervical del implante en la preservación de hueso marginal perimplantario: Una revisión sistemática. Int. $J$. Odontostomat., 9(1):119-127, 2015

Tabla I. Descripción de la estrategia de búsqueda PICO (Patient or Population, Intervention, Control or Comparison y Outcome).

\begin{tabular}{|c|c|c|}
\hline \multicolumn{3}{|c|}{$\begin{array}{l}\text { ESTRATEGIA SISTEMÁTICA DE BÚSQUEDA } \\
\text { Pregunta dirigida: ¿ES el diseño del cuello del }\end{array}$} \\
\hline \multicolumn{3}{|c|}{ Estrategia de Búsqueda } \\
\hline Problema & $\begin{array}{l}\text { Diseño de la región cervical del } \\
\text { implante }\end{array}$ & $\begin{array}{l}\text { \#1 dental implants AND neck design } \\
\text { \#2 dental implants AND neck aesthetics }\end{array}$ \\
\hline Intervención & Implantología Oral & \\
\hline Comparación & $\begin{array}{l}\text { Sistemas PS vs. no PS, microespiras } \\
\text { vs. no microespiras, nivel de colocación } \\
\text { del cuello }\end{array}$ & $\begin{array}{l}\text { \#3dental implants AND machined collar } \\
\text { \#4 dental implants AND platform switching } \\
\text { \#5 dental implants AND neck microthread }\end{array}$ \\
\hline Outcome & $\begin{array}{l}\text { Estabilidad del Hueso marginal } \\
\text { perimplantario }\end{array}$ & \#6 dental implants AND neck bone resorption \\
\hline \multicolumn{3}{|c|}{ Bases de datos } \\
\hline Electrónicas & \multicolumn{2}{|l|}{ Pubmed y Cochrane } \\
\hline Revistas & \multicolumn{2}{|c|}{$\begin{array}{l}\text { International Journal of Oral and maxillofacial Implants, Clinical Oral Implants Research, } \\
\text { Journal of Clinical Periodontology }\end{array}$} \\
\hline \multicolumn{3}{|c|}{ Criterios de Selec ción } \\
\hline \multirow[t]{2}{*}{ Inclusión } & \multicolumn{2}{|c|}{$\begin{array}{l}\text { Estudios Clínicos Controlados y aleato rizados, estudios clínicos prospectivos y } \\
\text { retrospectivos, revisiones sistemáticas }\end{array}$} \\
\hline & \multicolumn{2}{|c|}{ Periodos de seguimiento después de la carga iguales o mayores a un año } \\
\hline \multirow[t]{4}{*}{ Exclusión } & \multicolumn{2}{|l|}{ Series de casos o a propósito de un caso } \\
\hline & \multirow{2}{*}{\multicolumn{2}{|c|}{$\begin{array}{l}\text { Estudios in vitro, modelos numéricos o en animales } \\
\text { Estudios en los que no se indicaban mediciones del hueso marginal o con datos } \\
\text { imcompletos }\end{array}$}} \\
\hline & & \\
\hline & \multicolumn{2}{|l|}{ Periodo de publicación superior a 10 años } \\
\hline
\end{tabular}

\section{RESULTADOS}

La Tabla II muestra los resultados de la búsqueda inicial, que dio lugar a un total de 445 artículos, de los cuales, tras realizar una revisión por título, se seleccionaron 45 artículos. Una vez estudiados, se incluyeron un total de 16 estudios que cumplieron los criterios de inclusión y exclusión anteriormente especificados. La fecha de éstos osciló entre los años 2005 y 2014, siendo la mayoría de ellos posteriores a 2009. En estos trabajos se encontraron diferentes propuestas en cuanto a diseños del módulo de la cresta del implante con el objetivo de favorecer el mantenimiento óseo marginal. Entre ellos se pueden diferenciar principalmente los sistemas de cuello pulido, más o menos alto, y los sistemas que tratan la superficie del implante en su totalidad. Asimismo, algunas marcas añaden microespiras en esta zona cervical con el mismo objetivo. Finalmente, en lo que respecta al macrodiseño, destaca el platform-switching.

En base a esto, los estudios se clasificaron por el tipo de configuración del cuello del implante y el tipo de estudio (Tabla III). Ocho artículos fueron incluidos en la configuración de cuello mecanizado, 5 en cuello tratado, 9 incluyeron implantes con microespiras en

Tabla II. Resultados búsqueda inicial.

\begin{tabular}{lcccc}
\hline \multirow{2}{*}{ Palabras clave } & \multicolumn{2}{c}{ Pubmed } & \multicolumn{2}{c}{ Cochrane } \\
\cline { 2 - 4 } & Totales & Incluidos & Totales & Incluidos \\
\hline Dental Implants AND Neck design & 167 & 10 & 0 & 0 \\
Dental Implants AND Neck Bone Resorption & 73 & 2 & 0 & 0 \\
Dental Implants AND machined collar & 19 & 0 & 0 & 0 \\
Dental Implants AND Platform Switching & 132 & 3 & 3 & 1 \\
Dental Implants AND Neck Aesthetics & 33 & 0 & 2 & 0 \\
Dental Implants AND Neck microthread & 21 & 0 & 0 & 0 \\
\hline
\end{tabular}


DIÉGUEZ PEREIRA, M.; SANTAMARÍA ARRIETA G.; FERNÁNDEZ GONZÁLEZ, F. J.; VEGA ÁLVAREZ, J. A.; BELLANCO PINTA, I.; MARTíN BLANCO N. \& BRIZUELA VELASCO. A. Influencia del diseño micro y macroscópico de la región cervical del implante en la preservación de hueso marginal perimplantario: Una revisión sistemática. Int. J. Odontostomat., 9(1):119-127, 2015.

Tabla III. Modificaciones del cuello del implante para la preservación del hueso marginal de los diferentes estudios incluidos.

\begin{tabular}{|c|c|c|c|}
\hline $\begin{array}{l}\text { Método de } \\
\text { preservación ósea }\end{array}$ & ECAs & ECPs & ECRs \\
\hline \multirow[t]{4}{*}{ Cuello mecanizado } & Peñarrocha-Diago et al. (2013) & Van de Velde et al. (2010) & Heinemann et al. (2009) \\
\hline & den Hartog et al. (2011) & Bratu et al. (2009) & --- \\
\hline & Tan et al. (2011) & Nickenig et al. (2009) & --- \\
\hline & Shin et al. (2006) & - +- & --- \\
\hline \multirow[t]{4}{*}{ Cuello tratado } & Kang et al. (2012) & Van de Velde et al. (2010) & --- \\
\hline & Shin et al. (2006) & --- & --- \\
\hline & Trammel et al. (2009) & --- & --- \\
\hline & Enkling et al. (2013) & --- & --- \\
\hline \multirow[t]{4}{*}{ Microespiras } & Peñarrocha-Diago et al. (2013) & Van de Velde et al. (2010) & Heineman et al. (2009) \\
\hline & den Hartog et al. (2011) & Kim et al. (2010) & --- \\
\hline & Kang et al. (2012) & Bratu et al. (2009) & --- \\
\hline & Shin et al. (2006) & Nickenig et al. (2009) & --- \\
\hline Diseño festoneado & den Hartog et al. (2011) & --- & --- \\
\hline Platform Switching & Trammel et al. (2009) & --- & --- \\
\hline & Enkling et al. (2013) & --- & --- \\
\hline $\begin{array}{l}\text { Modificaciones de } \\
\text { superficie }\end{array}$ & Shin et al. (2006) & --- & --- \\
\hline
\end{tabular}

ECAs= estudios clínicos aleatorizados; ECPs= estudios clínicos prospectivos; ECRs= estudios clínicos retrospectivos.

su zona cervical como variable a estudiar, 1 artículo con diseño festoneado, 2 con platform-switching y 1 en el que se utilizaron implantes de superficie Friadent Plus (tratamiento no especificado), implantes con tratamiento por sustracción con chorreado de partículas de fosfato de calcio e implantes con superficie SLA.

En la Tabla IV se analiza además su nivel evidencia según CEBM de Oxford, y los riesgos de sesgo de los ECAs según la Herramienta Cochrane, se incluyeron un total de: 4 revisiones sistemáticas, 7 estudios clínicos aleatorizados (ECA), 4 estudios clínicos prospectivos (ECPs) y 1 estudio clínico retrospectivo (ECR)

Se contabilizaron un total de 477 pacientes y 1069 implantes colocados. El rango de pacientes en cada estudio fue de 10-93 (Media 39,75; desviación estándar 27,03) y el de implantes colocados de 24195 (Media 89,08; desviación estándar 56,86). Los períodos de observación variaron entre 12 y 34 meses (Media 16,18; desviación estándar 7,54), siempre desde la colocación de la prótesis.

Para llevar a cabo las mediciones de los cambios producidos en el hueso periimplantario, casi todos los autores emplearon radiografías intraorales calibradas, mientras que Nickenig et al. (2009) y Enkling et al. (2013) usaron ortopantomografías. Las pérdidas de hueso marginal oscilaron entre 0,05 y $1,52 \mathrm{~mm}$ entre los distintos diseños del módulo crestal del implante (Tabla V).

\section{DISCUSIÓN}

Debido a la gran heterogeneidad existente entre todos los estudios analizados (tipo de implante, protocolo quirúrgico, control, y tiempos de observación), no fue posible la realización de un análisis estadístico de los datos.

Respecto al momento de la toma de radiografías, algunos autores (Peñarrocha et al., 2013; den Hartog et al., 2011; Tan et al., 2011; Shin et al., 2006; Van de Velde et al., 2010; Bratu et al., 2009; Heinemann et al., 2009) no la realizan en dos momentos que pueden considerarse clave, como son la colocación del implante y la conexión de la prótesis. Es por ello, que en estos casos, no es posible determinar si la pérdida de hueso es consecuencia de la remodelación ósea post-quirúrgica, de la formación de un nuevo espacio biológico al conectar la prótesis o como resultado de la carga.

Además, se considera que las pérdidas iniciales de hueso se focalizan principalmente en la pared bucal (Misch, 1992), con lo cual, ni con radiografías periapicales calibradas ni con ortopantomografías po- 
DIÉGUEZ PEREIRA, M.; SANTAMARÍA ARRIETA G.; FERNÁNDEZ GONZÁLEZ, F. J.; VEGA ÁLVAREZ, J. A.; BELLANCO PINTA, I.; MARTÍN BLANCO N. \& BRIZUELA VELASCO. A. Influencia del diseño micro y macroscópico de la región cervical del implante en la preservación de hueso marginal perimplantario: Una revisión sistemática. Int. $J$. Odontostomat., 9(1):119-127, 2015

Tabla IV. Estudios incluidos según Centro de Medicina Basada en la Evidencia (CEBM) y riesgo de sesgo según Herramienta Cochrane de ECAs (estudios clínicos aleatorizados).

\begin{tabular}{|c|c|c|c|c|}
\hline $\begin{array}{l}\text { Tipo de } \\
\text { estudio }\end{array}$ & $\begin{array}{l}\text { Nivel de } \\
\text { evidencia } \\
\text { CEBM } \\
\text { Oxford }\end{array}$ & $\begin{array}{l}\text { Grado de } \\
\text { recomendación }\end{array}$ & Estudio & Riego de sesgo según herramienta Cochrane \\
\hline \multirow[t]{7}{*}{7 ECAs } & $1 \mathrm{~b}$ & A & $\begin{array}{l}\text { Peñarrocha-Diago et } \\
\text { al. (2013) }\end{array}$ & - Sesgo de realización. No se puede cegar al operador. \\
\hline & & & $\begin{array}{l}\text { den Hartog et al. } \\
\text { (2011) }\end{array}$ & $\begin{array}{l}\text { - Sesgo de realización. No se puede cegar al operador. } \\
\text { - Sesgo de detección. No se puede cegar al evaluador. } \\
\text { - Sesgo de notificación. No se toma rx en la colocación } \\
\text { de la prótesis. }\end{array}$ \\
\hline & & & Kang et al. (2012) & $\begin{array}{l}\text { - Sesgo de selección. No explica método de } \\
\text { aleatorización. } \\
\text { - Sesgo de realización. No se puede cegar al operador. } \\
\text { - Sesgo de detección. No se puede cegar al evaluador. }\end{array}$ \\
\hline & & & Tan et al. (2011) & $\begin{array}{l}\text { - Sesgo de realización. No se puede cegar al operador. } \\
\text { - Sesgo de detección. No se puede cegar al evaluador. } \\
\text { - Sesgo de notificación. No se toma rx en la colocación } \\
\text { de la prótesis. }\end{array}$ \\
\hline & & & Trammel et al. (2009) & $\begin{array}{l}\text { - Sesgo de realización. No se puede cegar al operador. } \\
\text { - Sesgo de detección. No se puede cegar al evaluador. }\end{array}$ \\
\hline & & & Shin et al. (2006) & $\begin{array}{l}\text { - Sesgo de realización. No se puede cegar al operador. } \\
\text { - Sesgo de detección. No se puede cegar al evaluador. } \\
\text { - Sesgo de notificación. No se toma rx en la colocación } \\
\text { de la prótesis. }\end{array}$ \\
\hline & & & Enkling et al. (2013) & $\begin{array}{l}\text { - Sesgo de realización. No se puede cegar al operador. } \\
\text { - Sesgo de detección. No se puede cegar al evaluador. }\end{array}$ \\
\hline \multirow[t]{4}{*}{4 ECPs } & $2 b$ & B & $\begin{array}{l}\text { Van de Velde et al. } \\
\text { (2010) }\end{array}$ & --- \\
\hline & & & Kim et al. (2010) & --- \\
\hline & & & Bratu et al. (2009) & --- \\
\hline & & & Nickenig et al. (2009) & --- \\
\hline \multirow[t]{4}{*}{$4 \mathrm{RS}$} & $2 a$ & B & $\begin{array}{l}\text { Aloy-Prósper et al. } \\
\text { (2011) }\end{array}$ & --- \\
\hline & & & Bateli et al. (2011) & --- \\
\hline & & & Annibali et al. (2012) & --- \\
\hline & & & Strietzel et al. (2015) & --- \\
\hline $1 \mathrm{ECR}$ & 4 & C & $\begin{array}{l}\text { Heinemann et al. } \\
\text { (2009) }\end{array}$ & --- \\
\hline
\end{tabular}

ECAs= estudios clínicos aleatorizados; ECPs= estudios clínicos prospectivos; ECRs= estudios clínicos retrospectivos; RS= revisiones sistemáticas.

drían detectarse, ya que con este tipo de exploraciones sólo podremos medir con cierta precisión las alturas de las crestas óseas proximales, mesial y distal.

Hasta en 4 de los estudios incluidos (Peñarrocha et al.; Tan et al.; Shin et al.; Bratu et al.) se siguió un protocolo quirúrgico basado en la colocación subcrestal del cuello pulido, asumiendo de este modo una pérdi- da ósea por lo menos igual a la altura de la superficie sin tratar. Además, en ninguno de ellos se controló radiográficamente el hueso marginal periimplantario tras el tiempo de cicatrización, de forma que no es posible sacar conclusiones al respecto. Sin embargo, Berglundh et al. (2005), al controlar esta variable, demuestran que la pérdida ósea alrededor del cuello pulido se produce durante el propio proceso de 
DIÉGUEZ PEREIRA, M.; SANTAMARÍA ARRIETA G.; FERNÁNDEZ GONZÁLEZ, F. J.; VEGA ÁLVAREZ, J. A.; BELLANCO PINTA, I.; MARTÍN BLANCO N. \& BRIZUELA VELASCO. A. Influencia del diseño micro y macroscópico de la región cervical del implante en la preservación de hueso marginal perimplantario: Una revisión sistemática. Int. J. Odontostomat., 9(1):119-127, 2015.

Tabla V. Pérdida ósea marginal (POM) de cada tipo de implante utilizado.

\begin{tabular}{|c|c|c|c|c|}
\hline Estudio & $\begin{array}{l}\text { Muestra/n } \\
\text { implantes }\end{array}$ & Seguimiento & Tipo de Implante & POM \\
\hline $\begin{array}{l}\text { Peñarrocha et al. } \\
\text { (2013) }\end{array}$ & $18 / 141$ & 6 y 12 meses & $\begin{array}{l}\text { Mecanizado externa } 2,5 \\
\text { Microespiras interna }\end{array}$ & $\begin{array}{l}0,38 \pm 0,51 \\
0,12 \pm 0,17\end{array}$ \\
\hline $\begin{array}{l}\text { den Hartog et al. } \\
(2011)\end{array}$ & $93 / 279$ & 3 y 12 meses & $\begin{array}{l}\text { Pulido de } 1,5 \\
\text { Microespiras } \\
\text { Microespiras y } \\
\text { festoneado }\end{array}$ & $\begin{array}{l}1,19 \pm 0,82 \\
0,90 \pm 0,57 \\
2,01 \pm 0,77\end{array}$ \\
\hline Kang et al. (2011) & $20 / 40$ & $\begin{array}{c}\text { Prótesis y } 12 \\
\text { meses }\end{array}$ & $\begin{array}{l}\text { Microespiras } \\
\text { Macroespiras }\end{array}$ & $\begin{array}{l}0,15 \pm 0,14 \\
0,13 \pm 0,14\end{array}$ \\
\hline Tan et al. (2011) & $18 / 36$ & 6 y 12 meses & $\begin{array}{l}\text { Cuello pulido } 1,8 \\
\text { Cuello pulido } 2,8\end{array}$ & $\begin{array}{l}0,87 \\
1.31\end{array}$ \\
\hline Shin et al. (2006) & $68 / 107$ & 3,6 y 12 meses & $\begin{array}{l}\text { Cuello pulido } 2,8 \\
\text { Cuello tratado } \\
\text { Microespiras }\end{array}$ & $\begin{array}{l}1,32 \\
0,76 \\
0,18\end{array}$ \\
\hline $\begin{array}{l}\text { Trammell et al. } \\
\text { (2009) }\end{array}$ & $10 / 25$ & 12 y 24 meses & $\begin{array}{l}\text { Cuello tratado PM } \\
\text { Cuello tratado PS }\end{array}$ & $\begin{array}{l}1,19 \pm 0,58 \\
0,99 \pm 0,53\end{array}$ \\
\hline Enkling et al. (2013) & $25 / 50$ & $\begin{array}{c}8,12,25 \text { y } 38 \\
\text { meses }\end{array}$ & $\begin{array}{l}\text { Cuello tratado PM } \\
\text { Cuello tratado PS }\end{array}$ & $\begin{array}{l}0,74 \pm 0,57 \\
0,69 \pm 0,43\end{array}$ \\
\hline $\begin{array}{l}\text { Van de Velde et al. } \\
(2010)\end{array}$ & $39 / 195$ & 12 meses & $\begin{array}{l}\text { Cuello pulido } \\
\text { Cuello tratado } \\
\text { Microespiras }\end{array}$ & $\begin{array}{l}1,52 \pm 0,64 \\
0,80 \pm 0,98 \\
0,81 \pm 1,11\end{array}$ \\
\hline Kim et al. (2010) & $12 / 24$ & $\begin{array}{c}2^{a} \text { cir ugía, } \\
\text { carga, } 12 \text { meses }\end{array}$ & $\begin{array}{l}\text { Microespiras recto } \\
\text { Microespiras cónico }\end{array}$ & $\begin{array}{l}0,05 \pm 0,09 \\
0,07 \pm 0,14\end{array}$ \\
\hline Bratu et al. (2009) & $48 / 96$ & 4,6 y 12 meses & $\begin{array}{l}\text { Cuello pulido } 1 \mathrm{~mm} \\
\text { Microespiras }\end{array}$ & $\begin{array}{c}1,47 \pm 0,4 \\
0,69 \pm 0,25\end{array}$ \\
\hline $\begin{array}{l}\text { Nickenig et al. } \\
(2009)\end{array}$ & $34 / 133$ & $\begin{array}{l}\text { Carga, } 6 \text { y } 22 \\
\text { meses (media) }\end{array}$ & $\begin{array}{l}\text { Microespiras } \\
\text { Cuello pulido } 1,5\end{array}$ & $\begin{array}{c}0,50 \\
1,1\end{array}$ \\
\hline $\begin{array}{l}\text { Heinemann et al. } \\
(2009)\end{array}$ & $7 / 24$ & $\begin{array}{c}\text { Variables-hasta } \\
18 \text { meses }\end{array}$ & $\begin{array}{l}\text { Cuello pulido } 1 \mathrm{~mm} \\
\text { Cuello pulido } 1 \mathrm{~mm} \\
\text { interna } \\
\text { Microespiras } 1,6 \mathrm{~mm}\end{array}$ & $\begin{array}{l}\text { No diferencias } \\
\text { significativas }\end{array}$ \\
\hline
\end{tabular}

osteointegración. Sin tomar en consideración estas limitaciones, se podría inferir que en un plazo medio de un año, un diseño del cuello del implante con microespiras podría favorecer la conservación del hueso marginal periimplantario mejor que un cuello pulido, especialmente en los 6 primeros meses post-carga y de manera menos acusada en adelante. Esta circunstancia podría deberse a un mayor cerclaje del implante alrededor de la cresta ósea cortical, lo que aseguraría un menor nivel de micromovimientos durante la fase de osteointegración o a una mejora de las transferencias de carga al terreno de soporte. Sin embargo, los estudios de elementos finitos no presentan consenso a este respecto (Ferraz et al., 2012; Rahimi et al., 2009).

En cuanto al intercambio de plataforma encontramos también resultados diversos. Trammel et al. (2009), afirman que el intercambio de plataforma re- duce de forma considerable la pérdida de hueso periimplantario marginal. Sin embargo, Enkling et al., concluyen que no existen diferencias significativas entre ambos grupos $(<0,1 \mathrm{~mm})$.

En cuanto al manejo de los datos, Heinemann et al., en su estudio seleccionan 24 implantes de los 118 colocados sin especificar el motivo de su elección. Además cada uno de estos implantes tiene un período de seguimiento diferente, en un rango de 518 meses desde la colocación.

En este estudio también han sido incluidas 4 revisiones sistemáticas que presentan conclusiones dispares. Aloy-Prosper et al. (2009), concluyen que la incorporación de un diseño con microespiras a un cuello tratado (de superficie rugosa) supone una ventaja respecto a la conservación de hueso marginal. Sin embargo, Bateli et al. (2011), aseguran que son 
DIÉGUEZ PEREIRA, M.; SANTAMARÍA ARRIETA G.; FERNÁNDEZ GONZÁLEZ, F. J.; VEGA ÁLVAREZ, J. A.; BELLANCO PINTA, l.; MARTÍN BLANCO N. \& BRIZUELA VELASCO. A. Influencia del diseño micro y macroscópico de la región cervical del implante en la preservación de hueso marginal perimplantario: Una revisión sistemática. Int. $J$. Odontostomat., 9(1):119-127, 2015.

necesarios un mayor número de estudios para determinar el papel del diseño del módulo crestal del implante en la remodelación ósea marginal. En cuanto al macrodiseño, Annibali et al. (2012) y Strietzel et al. (2015), defienden que la técnica de intercambio de plataforma limita la reabsorción ósea, aunque debido a la heterogeneidad, los factores de confusión y los breves períodos de observación de los estudios, no se puede inferir una conclusión clara al respecto. Una posición apico-coronal yuxtaósea del cuello pulido de un implante, respecto a la cresta ósea receptora es crítico en lo que concierne a la conservación de hueso marginal durante el proceso de osteointegración y los 3 primeros meses de carga funcional. No es posible concluir una mejora en la conservación de hueso para ninguno de los diseños de cuello estudiados.

Son precisos un mayor número de estudios clínicos, con controles más precisos, muestras más grandes y períodos de seguimiento mayores, que estudien estos factores.

DIÉGUEZ PEREIRA, M.; SANTAMARÍA ARRIETA G.; FERNÁNDEZ GONZÁLEZ, F. J.; VEGA ÁlVAREZ, J. A.; BELLANCO PINTA, I.; MARTÍN BLANCO N. \& BRIZUELA VELASCO. A. Influence of the micro and macroscopic implant neck design in the preservation of marginal bone level: A systematic review. Int. J. Odontostomat., 9(1):119-127, 2015.

ABSTRACT: The aim of this systematic review was to assess whether different implant neck designs (polished or coated, with or without platform switching, either with or without microthreads) influence marginal bone resorption. Asystematic review was conducted following the PICO methodology of the Centre for Evidence-Based Medicine of Oxford University. The question to the achievement of objectives was: Is the design of the implant neck a critical factor in the preservation of marginal bone levels? Specific MeSH keywords were used in the Pubmed and Cochrane databases. Three independent reviewers agreed on the studies ultimately included finding a concordance kappa index of 0.86 . Of these articles, acritical evaluation of the level of evidence was performed and also the risk of bias of the RCT using the Cochrane tool. Four hundred forty-five items were reviewed by title, eventually including a total of 16 full-text articles. Heterogeneity of these studies made impossible the performance of a meta-analysis. No evidence that a particular configuration of the implant neck was better than another in preserving the peri-implant marginal bone was found, and yet it seems that an apico-coronal position juxtaosseous of the smooth neck of the implant relative to the bone crest could be decisive. Not enough evidence was found on the effectiveness of different configurations of the cervical region of the implant in the periimplant marginal bone preservation. More randomized controlled trials are needed to assess long-term effects of such modifications. switching.

KEY WORDS: bone resorption, implant collar, implant neck, marginal bone loss, neck design, platform-

\section{REFERENCIAS BIBLIOGRÁFICAS}

Abrahamsson, I.; Berglundh, T.; Glantz, P. O. \& Lindhe, J. The mucosal attachment at different abutments. An experimental study in dogs. J. Clin. Periodontol., 25(9):7217, 1998.

Adell, R.; Eriksson, B.; Lekholm, U.; Branemark, P. I. \& Jemt, T. Long-term follow-up study of osseointegrated implants in the treatment of totally edentulous jaws. Int. J. Oral Maxillofac. Implants, 5(4):347-59, 1990.

Albrektsson, T.; Zarb, G.; Worthington, P. \& Eriksson, A. R. The long-term efficacy of currently used dental implants: a review and proposed criteria of success. Int. J. Oral Maxillofac. Implants, 1(1):11-25, 1986.

Aloy-Prósper, A.; Maestre-Ferrín, L.; Peñarrocha-Oltra, D. \& Peñarrocha-Diago, M. Marginal bone loss in relation to the implant neck surface: an update. Med. Oral Patol. Oral Cir. Bucal, 16(3):e365-8, 2011.
Annibali, S.; Bignozzi, I.; Cristalli, M. P.; Graziani, F.; La Monaca, G. \& Polimeni, A. Peri-implant marginal bone level: a systematic review and meta-analysis of studies comparing platform switching versus conventionally restored implants. J. Clin. Periodontol., 39(11):1097-113, 2012.

Assunção, W. G.; Gomes, E. A.; Rocha, E. P. \& Delben, J. A. Three-dimensional finite element analysis of vertical and angular misfit in implant-supported fixed prostheses. Int. J. Oral Maxillofac. Implants, 26(4):788-96, 2011.

Bateli, M.; Att, W. \& Strub, J. R. Implant neck configurations for preservation of marginal bone level: a systematic review. Int. J. Oral Maxillofac. Implants, 26(2):290-303, 2011.

Berglundh, T.; Abrahamsson, I. \& Lindhe, J. Bone reactions to longstanding functional load at implants: an experi- 
DIÉGUEZ PEREIRA, M.; SANTAMARÍA ARRIETA G.; FERNÁNDEZ GONZÁLEZ, F. J.; VEGA ÁLVAREZ, J. A.; BELLANCO PINTA, I.; MARTÍN BLANCO N. \& BRIZUELA VELASCO. A. Influencia del diseño micro y macroscópico de la región cervical del implante en la preservación de hueso marginal perimplantario: Una revisión sistemática. Int. $J$. Odontostomat., 9(1):119-127, 2015.

mental study in dogs. J. Clin. Periodontol., 32(9):925-32, 2005 .

Branemark, P. I.; Hansson, B. O.; Adell, R.; Breine, U.; Lindström, J.; Hallén, O. \& Ohman, A. Osseointegrated implants in the treatment of the edentulous jaw. Experience from a 10-year period. Scand. J. Plast. Reconstr. Surg. Suppl., 16:1-132, 1977.

Bratu, E. A.; Tandlich, M. \& Shapira, L. A rough surface implant neck with microthreads reduces the amount of marginal bone loss: a prospective clinical study. Clin. Oral Implants Res., 20(8):827-32, 2009.

Chang, C. L.; Chen, C. S. \& Hsu, M. L. Biomechanical effect of platform switching in implant dentistry: a three-dimensional finite element analysis. Int. J. Oral Maxillofac. Implants, 25(2):295-304, 2010.

den Hartog, L.; Meijer, H. J.; Stegenga, B.; Tymstra, N.; Vissink, A. \& Raghoebar, G. M. Single implants with different neck designs in the aesthetic zone: a randomized clinical trial. Clin. Oral Implants Res., 22(11):1289-97, 2011.

Enkling, N.; Jöhren, P.; Katsoulis, J.; Bayer, S.; Jervøe-Storm, P. M.; Mericske-Stern, R. \& Jepsen, S. Influence of platform switching on bone-level alterations: a three-year randomized clinical trial. J. Dent. Res., 92 (12 Suppl.):139S-45S, 2013.

Ferraz, C. C.; Anchieta, R. B.; de Almeida, E. O.; Freitas, A. C. Jr.; Ferraz, F. C.; Machado, L. S. \& Rocha, E. P. Influence of microthreads and platform switching on stress distribution in bone using angled abutments. J. Prosthodont. Res., 56(4):256-63, 2012.

Grunder, U.; Gracis, S. \& Capelli, M. Influlnfluence of the 3-D bone-to-implant relationship on esthetics. Int. J. Periodontics Restorative Dent., 25(2):113-9, 2005.

Heinemann, F.; Bourauel, C.; Hasan, I. \& Gedrange, T. Influence of the implant cervical topography on the crestal bone resorption and immediate implant survival. J. Physiol. Pharmacol., 60 (Suppl. 8):99-105, 2009.

Hermann, J. S.; Buser, D.; Schenk, R. K.; Schoolfield, J. D. \& Cochran, D. L. Biologic Width around one- and two-piece titanium implants. Clin. Oral Implants Res., 12(6):559-71, 2001.

Isidor, F. Loss of osseointegration caused by occlusal load of oral implants. A clinical and radiographic study in monkeys. Clin. Oral Implants Res., 7(2):143-52, 1996.

Jadad, A. R.; Moore, R. A.; Carroll, D.; Jenkinson, C.; Reynolds, D. J.; Gavaghan, D. J. \& McQuay, H. J. Assessing the quality of reports of randomized clinical trials: is blinding necessary? Control. Clin. Trials, 17(1):1-12, 1996.
Kang, Y. I.; Lee, D. W.; Park, K. H. \& Moon, I. S. Effect of thread size on the implant neck area: preliminary results at 1 year of function. Clin. Oral Implants Res., 23(10):114751,2012

Lang, N. P.; Berglundh, T. \& Working Group 4 of Seventh European Workshop on Periodontology. Periimplant diseases:where are we now?--Consensus of the Seventh European Workshop on Periodontology. J. Clin. Periodontol., 38 Suppl. 11:178-81, 2011.

Lazzara, R. J. \& Porter, S. S. Platform switching: a new concept in implant dentistry for controlling postrestorative crestal bone levels. Int. J. Periodontics Restorative Dent., 26(1):917, 2006.

Maeda, Y.; Miura, J.; Taki, I. \& Sogo, M. Biomechanical analysis on platform switching: is there any biomechanical rationale? Clin. Oral Implants Res., 18(5):581-4, 2007.

Misch, C. E. Implant success or failure: Clinical assessment. In: Misch, C. E. (Ed.). Contemporary Implant Dentistry. St. Louis, Mosby, 1992. pp.29-42.

Miyata, T.; Kobayashi, Y.; Araki, H.; Motomura, Y. \& Shin, K. The influence of controlled occlusal overload on periimplant tissue: a histologic study in monkeys. Int. J. Oral Maxillofac. Implants, 13(5):677-83, 1998.

Miyata, T.; Kobayashi, Y.; Araki, H.; Ohto, T. \& Shin, K. The influence of controlled occlusal overload on peri-implant tissue. Part 3: A histologic study in monkeys. Int. J. Oral Maxillofac. Implants, 15(3):425-31, 2000.

Nickenig, H. J.; Wichmann, M.; Schlegel, K. A.; Nkenke, E. \& Eitner, S. Radiographic evaluation of marginal bone levelsadjacent to parallel-screw cylinder machined-neck implants and rough-surfaced microthreaded implants using digitized panoramic radiographs. Clin. Oral Implants Res., 20(6):550-4, 2009.

Peñarrocha-Diago, M. A.; Flichy-Fernández, A. J.; AlonsoGonzález, R.; Peñarrocha-Oltra, D.; Balaguer-Martínez, J. \& Peñarrocha-Diago, M. Influence of implant neck design and implant-abutment connection type on periimplant health. Radiological study. Clin. Oral Implants Res., 24(11):1192-200, 2013.

Rahimi, A.; Bourauel, C.; Jager, A.; Gedrange, T. \& Heinemann, F. Load transfer by fine threading the implant neck--a FEM study. J. Physiol. Pharmacol., 60 (Suppl. 8):107-12, 2009.

Rungsiyakull, C.; Rungsiyakull, P.; Li, Q.; Li, W. \& Swain, M. Effects of occlusal inclination and loading on mandibular bone remodeling: a finite element study. Int. J. Oral Maxillofac. Implants, 26(3):527-37, 2011.

Shin, Y. K.; Han, C. H.; Heo, S. J.; Kim, S. \& Chun, H. J. 
DIÉGUEZ PEREIRA, M.; SANTAMARÍA ARRIETA G.; FERNÁNDEZ GONZÁLEZ, F. J.; VEGA ÁLVAREZ, J. A.; BELLANCO PINTA, I.; MARTÍN BLANCO N. \& BRIZUELA

VELASCO. A. Influencia del diseño micro y macroscópico de la región cervical del implante en la preservación de hueso marginal perimplantario: Una revisión sistemática. Int. $J$. Odontostomat, 9(1):119-127, 2015

Radiographic evaluation of marginal bone level around implants with different neck designs after 1 year. Int. J. Oral Maxillofac. Implants, 21(5):789-94, 2006.

Strietzel, F. P.; Neumann, K. \& Hertel, M. Impact of platform switching on marginal peri-implant bone-level changes. A systematic review and meta-analysis. Clin. Oral Implants Res., 26(3):342-58, 2015.

Tan, W. C.; Lang, N. P.; Schmidlin, K.; Zwahlen, M. \& Pjetursson, B. E. The effect of different implant neck configurations on soft and hard tissue healing: a randomized-controlled clinical trial. Clin. Oral Implants Res., 22(1):14-9, 2011.

Tomasi, C.; Sanz, M.; Cecchinato, D.; Pjetursson, B.; Ferrus, J.; Lang, N. P. \& Lindhe, J. Bone dimensional variations at implants placed in fresh extraction sockets: a multilevel multivariate analysis. Clin. Oral Implants Res., 21(1):306, 2010.

Trammell, K.; Geurs, N. C.; O'Neal, S. J.; Liu, P. R.; Haigh, S. J.; McNeal, S.; Kenealy, J. N. \& Reddy M. S. A prospective, randomized, controlled comparison of platform-switched and matched-abutment implants in short-span partial denture situations. Int. J. Periodontics Restorative Dent., 29(6):599-605, 2009.

Van de Velde, T.; Collaert, B.; Sennerby, L. \& De Bruyn, H. Effect of implant design on preservation of marginal bone in the mandible. Clin. Implant Dent. Relat. Res., 12(2):13441, 2010.
Dirección para Correspondencia:

Dr. Felipe J. Fernández-González

Profesor Posgrado de Ortodoncia y Ortopedia Dentofacial Universidad de Oviedo

Oviedo

ESPAÑA

Email: fjvazquezvega@gmail.com

Recibido: 11-09-2014

Aceptado: 04-03-2015 